\title{
Mechanical Verification of Transactional Memories with Non-transactional Memory Accesses ${ }^{\star}$
}

\author{
Ariel Cohen ${ }^{1}$, Amir Pnueli ${ }^{1}$, and Lenore D. Zuck ${ }^{2}$ \\ ${ }^{1}$ New York University, \\ \{arielc, amir\}@es.nyu.edu \\ ${ }^{2}$ University of Illinois at Chicago, \\ lenoreacs.uic.edu
}

\begin{abstract}
Transactional memory is a programming abstraction intended to simplify the synchronization of conflicting memory accesses (by concurrent threads) without the difficulties associated with locks. In a previous work we presented a formal framework for proving that a transactional memory implementation satisfies its specifications and provided with model checking verification of some using small instantiations. This paper extends the previous work to capture nontransactional accesses to memory, which occurs, for example, when using legacy code. We provide a mechanical proof of the soundness of the verification method, as well as a mechanical verification of a version of the popular TCC implementation that includes non-transactional memory accesses. The verification is performed by the deductive temporal checker TLPVS.
\end{abstract}

\section{Introduction}

Transactional Memory [5] is a simple solution for coordinating and synchronizing concurrent threads that access the same memory locations. It transfers the burden of concurrency management from the programmers to the system designers and enables a safe composition of scalable applications, we well as efficiently utilizes the multiple cores. Multicore and many-core processors, which require concurrent programs in order to gain a full advantage of the multiple number of processors, has become the mainstream architecture for microprocessor chips and thus many new transactional memory implementations have been proposed recently (see [9] for an excellent survey).

A transactional memory (TM) receives requests from clients and issues responses. The requests are usually part of a transaction that is a sequence of operations starting with a request to open a transaction, followed by a sequence of read/write requests, followed by a request to commit (or abort). The TM responds to requests. When a transaction requests a successful "commit," all of its effects are stored in the memory. If a transaction is aborted (by either issuing an abort request or when TM detects that it should be aborted) all of its effects are removed. Thus, a transaction is a sequence of atomic operations, either all complete successfully and all its write operations update the memory, or none completes and its write operations do not alter the memory. In

\footnotetext{
* This research was supported in part by ONR grant N00014-99-1-0131 and NSF grants CCF0742686 and CNS-0720525.
} 
addition, committed transaction should be serializable - the sequence of operations belonging to successful transactions should be such that it can be reordered (preserving the order of operations in each transaction) so that the operation of each transaction appear consecutive, and a "read" from any memory location returns the value of the last "write" to that memory location.

TMs are often parameterized by their properties. These may include the conflicts they are to avoid, when are the conflicts detected, how they are resolved, when is the memory updated, whether transactions can be nested, etc. (see [9] for a list of such properties). Each set of parameters defines a unique set of sequences of events that can occur is a TM so to guarantee atomicity and serializability. We refer to the set of sequences of events allowed by a TM as its sspecifications. A particular implementation does not necessarily generate all allowed sequences, but should only generate allowed sequences. The topic of this paper (as well as [3]) is to formally verify that a TM implementation satisfies its specification that is uniquely defined by its parameters.

Such parameters were given in [12]'s widely-cited paper, which was the first to characterize transactional memory in a way that captured and clarified the many semantic distinctions among the most popular implementations of TMs. Scott's ([12]) approach is to begin with classical notions of transactional histories and sequential specifications, and to introduce two important notions. The first is a conflict function which specifies when two overlapping (concurrent) transactions cannot both succeed (a safety condition). The second is an arbitration function which specifies which of two transactions must fail (a liveness condition). Scott's work went a long way towards clarification of the semantics of TMs, but did not facilitate mechanical verification of implementations.

The work in [3] (co-authored by the authors of this paper) took a first step towards modeling TMs, accordingly to [12]'s parameters, so to as allow for mechanical verification of their implementations. There, a specification of a TM is represented by a fair state machine that is parameterized by a set of admissible interchanges - a set of rules specifying when a pair of consecutive operations in a sequence of transactional operations can be safely swapped without introducing or removing a conflict. All the conflicts described in [12] can be cast as admissible sets. The specification machine takes a stream of transactional requests as inputs, and outputs a serializable sequence of the input requests and their responses. The fairness is used to guarantee that each transaction is eventually closed (committed or aborted) and, if committed, appears in the output. Some proof rules are given to show that a TM implementation satisfies its specification. The applicability of the approach is demonstrated on several well-known TM implementations. Small instantiations of each of the case study were shown to specify their specification using the model checker TLC [8].

This paper extends the work of [3] in two directions. The first is to add another parameter to the system - non-transactional memory accesses. Unlike their transactional counterparts, non-transactional accesses cannot be aborted. While atomicity and serializability requirements remain, where a non-transaction operation is cast as a singleton, successfully committed, transaction. The second direction is a framework that allows for a mechanical formal verification that TM implementations satisfy their specifications. The tool we use is TLPVS [11], which embeds temporal logic and its deductive frame-work within the theorem prover PVS [10]. Using TLPVS entailed some changes to 
the [3] proof rules that establish that an implementation indeed refines its specification. In fact, the rule presented here is more general than its predecessor. Using TLPVS also entailed restricting to interchange rules that can be described by temporal logics (which still covers all of [12]'s conflicts). For simplicity, we chose to restrict to interchanges whose temporal description uses only past temporal operators (i.e., depend only on the history leading to the interchange), which rules out [12]'s mixed invalidation conflict.

We make here a strong assumption on non-transactional accesses, namely, that the transactional memory is aware that non-transactional accesses, as soon as they occur. While the TM cannot abort such accesses, it may use them in order to abort transactions that are under its control. It is only with such or similar assumption that total consistency or coherence can be maintained.

We demonstrate the new framework by presenting TLPVS proofs that some TM implementations with non-transactional accesses satisfy their specifications, given an admissible interchange.

To the best of our knowledge, the work presented here is the first to employ a theorem prover for verifying correctness of transactional memories and the first to formally verify an implementation that handles non-transactional memory accesses.

The rest of the paper is organized as follows: Section 2 provides preliminary definitions related to transactional memory, and defines the concept of admissible interchanges. Section 3 provides a specification model of a transactional memory. Section 4 discusses a proof rule for verifying implementations. Section 5 presents a simple implementation of transactional memory that handles non-transactional memory accesses. Section 6 shows how to apply deductive verification using TLPVS to verify this implementation. Section 7 provides some conclusions and open problems.

\section{Transactional Sequences and Interchanges}

We extend the [3] to support non-transactional memory accesses and separate each action into a request/response pair, as well as give a temporal definition for interchanges.

\subsection{Transactional Sequences}

Assume $n$ clients that direct requests to a memory system, denoted by memory. For every client $p$, let the set of non-transactional invocations by client $p$ consists of:

- $\iota R_{p}^{n t}(x)$ - A non-transactional request to read from address $x \in \mathbb{N}$.

- $\iota W_{p}^{n t}(y, v)$ - A non-transactional request to write value $v \in \mathbb{N}$ to address $y \in \mathbb{N}$.

Let the set of transactional invocations by client $p$ consists of:

$-\iota \triangleleft_{p}-$ An open transaction request.

- $\iota R_{p}^{t}(x)-\mathrm{A}$ transactional read request from address $x \in \mathbb{N}$.

- $\iota W_{p}^{t}(y, v)-\mathrm{A}$ transactional request to write the value $v \in \mathbb{N}$ to address $y \in \mathbb{N}$.

- $\downarrow_{p}$ - A commit transaction request.

$-\iota p-$ An abort transaction request. 
The memory provides a response for each invocation. Erroneous invocations (e.g., a $\iota \varangle_{p}$ while client $p$ has a pending transaction) are responded by the memory returning an error flag err. Non-erroneous invocations, except for $\iota R^{t}$ and $\iota R^{n t}$ are responded by the memory returning an acknowledgment $a c k$. Finally, for non-erroneous $\iota R_{p}^{t}(x)$ and $\iota R_{p}^{n t}(x)$ the memory returns the (natural) value of the memory at location $x$. We assume that invocations and responses occur atomically and consecutively, i.e., there are no other operation that interleave an invocation and its response.

Let $E_{p}^{n t}:\left\{R_{p}^{n t}(x, u), W_{p}^{n t}(x, v)\right\}$ be the set of non-transactional observable events, $E_{p}^{t}:\left\{\boldsymbol{\triangleleft}_{p}, R_{p}^{t}(x, u), W_{p}^{t}(x, v), \nabla_{p}, \psi_{p}\right\}$ be the set of transactional observable events and $E_{p}=E^{n t} \cup E^{t}$, i.e. all events associated with client $p$. We consider as observable events only requests that are accepted, and abbreviate the pair (invocation, non-err response) by omitting the $\iota$-prefix of the invocation. Thus, $W_{p}^{t}(x, v)$ abbreviates $\iota W_{p}^{t}(x, v), a c k_{p}$. For read actions, we include the value read, that is, $R_{p}^{t}(x, u)$ abbreviates $\iota R^{t}(x), \rho R(u)$. When the value written/read has no relevance, we write the above as $W_{p}^{t}(x)$ and $R_{p}^{t}(x)$. When both values and addresses are of no importance, we omit the addresses, thus obtaining $W_{p}^{t}$ and $R_{p}^{t}$ (symmetric abbreviations and shortcuts are used for the non-transactional observable events). The output of each action is its relevant observable event when the invocation is accepted, and undefined otherwise. Let $E$ be the set of all observable events over all clients, i.e., $E=\bigcup_{p=1}^{n} E_{p}$ (similarly define $E^{n t}$ and $E^{t}$ to be the set of all non-transactional and the set of all transactional observable events, respectively).

Let $\sigma: e_{0}, e_{1}, \ldots, e_{k}$ be a finite sequence of observable $E$-events. We say that the sequence $\hat{\sigma}$ over $E^{t}$ is $\sigma^{\prime}$ 's transactional sequence, where $\hat{\sigma}$ is obtained from $\sigma$ by replacing each $R_{p}^{n t}$ and $W_{p}^{n t}$ by $\boldsymbol{\iota}_{p} R_{p}^{t} \boldsymbol{}_{p}$ and $\boldsymbol{\iota}_{p} W_{p}^{t} \boldsymbol{}_{p}$, respectively. That is, each non-transactional event of $\sigma$ is transformed into a singleton committed transaction in $\hat{\sigma}$. The sequence $\sigma$ is called a well-formed transactional sequence (TS for short) if the following all hold:

1. For every client $p$, let $\left.\hat{\sigma}\right|_{p}$ be the sequence obtained by projecting $\hat{\sigma}$ onto $E_{p}^{t}$. Then $\left.\hat{\sigma}\right|_{p}$ satisfies the regular expression $T_{p}^{*}$, where $T_{p}$ is the regular expression $\iota_{p}\left(R_{p}^{t}+\right.$ $\left.W_{p}^{t}\right)^{*}\left({ }_{p}+{ }_{p}\right)$. For each occurrence of $T_{p}$ in $\left.\hat{\sigma}\right|_{p}$, we refer to its first and last elements as matching. The notion of matching is lifted to $\hat{\sigma}$ itself, where $\boldsymbol{\iota}_{p}$ and $>_{p}\left(\right.$ or $\left.\psi_{p}\right)$ are matching if they are matching in $\left.\hat{\sigma}\right|_{p}$;

2. The sequence $\hat{\sigma}$ is locally read-write consistent: for any subsequence of $\hat{\sigma}$ of the form $\left\langle W_{p}^{t}(x, v) \eta R_{p}^{t}(x, u)\right\rangle$ where $\eta$ contains no ${ }_{p}$, ${ }_{p}$, or $W_{p}^{t}(x)$ events, $u=v$.

We denote by $\mathcal{T}$ the set of all well-formed transactional sequences, and by $\operatorname{pref}(\mathcal{T})$ the set of $\mathcal{T}$ 's prefixes. Note that the requirement of local read-write consistency can be enforced by each client locally. To build on this observation, we assume that, within a single transaction, there is no $R_{p}^{t}(x)$ following a $W_{p}^{t}(x)$, and there are no two reads or two writes to the same address. With these assumptions, the requirement of local read-write consistency is always (vacuously) satisfied. A TS $\sigma$ is atomic if:

1. $\hat{\sigma}$ satisfies the regular expression $\left(T_{1}+\cdots+T_{n}\right)^{*}$. That is, there is no overlap between any two transactions;

2. $\hat{\sigma}$ is globally read-write consistent: namely, for any subsequence $W_{p}^{t}(x, v) \eta$ $R_{q}^{t}(x, u)$ in $\hat{\sigma}$, where $\eta$ contains ${ }_{p}$, which is not preceded by ${ }_{p}$, and contains no event $W_{k}^{t}(x)$ followed by event $\boldsymbol{}_{k}$, it is the case that $u=v$. 


\subsection{Interchanging Events}

The notion of a correct implementation is that every TS can be transformed into an atomic TS by a sequence of interchanges which swap two consecutive events. This definition is parameterized by the set $\mathcal{A}$ of admissible interchanges which may be used in the process of serialization. Rather than attempt to characterize $\mathcal{A}$, we choose to characterize its complement $\mathcal{F}$, the set of forbidden interchanges. The definition here differs from the one in [3] in two aspects: There, in order to characterize $\mathcal{F}$, we allowed arbitrary predicates over the TS, here, we restrict to temporal logic formulae. Also, while [3] allowed swaps that depend on future events, here we restrict to swaps whose soundness depends only on the history leading to them. This restriction simplifies the verification process, and is the one used in all TM systems we are aware of. Note that it does not allow to express [12]'s mixed invalidation conflict. In all our discussions, we assume strict serializability which implies that while serializing a TS, the order of committed transactions has to be preserved.

Consider a temporal logic over $E$ using the past operators $\ominus$ (previously), $\diamond$ (sometimes in the past), and $\mathcal{S}$ (since). Let $\sigma$ be a prefix of a well-formed TS over $E^{t}$ (i.e., $\sigma=\hat{\sigma}$ ). We define a satisfiability relation $\models$ between $\sigma$ and a temporal logic formula $\varphi$ so that $\sigma \models \varphi$ if at the end of $\sigma, \varphi$ holds. (The more standard notation is $(\sigma,|\sigma|-1) \models \varphi$, but since we always interpret formulae at the end of sequences we chose the simplified notation.)

Some of the restrictions we place in $\mathcal{F}$ are structural. For example, the formula $p \neq q \wedge \triangleright_{p} \wedge \ominus \triangleright_{q}$ forbids the interchange of closures of transactions belonging to different clients. This guarantees the strictness of the serializability process. Similarly, the restriction $u_{p} \wedge \ominus v_{p}$, where $u_{p}, v_{p} \in E_{p}$, forbids the interchanges of two events belonging to the same client. Other formulas may guarantee the absence of certain conflicts. For example, following [12], a lazy invalidation conflict occurs when committing one transaction may invalidate a read of another, i.e., if for some transactions $T_{p}$ and $T_{q}$ and some memory address $x$, we have $R_{p}(x), W_{q}(x) \prec{ }_{q} \prec$ (where " $e_{i} \prec e_{j}$ " denotes that $e_{i}$ precedes $e_{j}$ ). Formally, the last two events in $\sigma$ cannot be interchanged when for some $p \neq q$,

$$
\sigma \models \triangleright_{q} \wedge \ominus\left(R_{p}(x) \wedge\left(\neg \triangleright_{q}\right) \mathcal{S} W_{q}(x)\right)
$$

Similarly, we express conflicts by TL formulae that determine, for any prefix of a TS (that includes only $E^{t}$ events), whether the two last events in the sequence can be safely interchanged without removing the conflict. For a conflict $c$, the formula that forbids interchanges that may remove instances of this conflict is called the maintaining formula for $c$ and is denoted by $m_{c}$. Thus, Formula 1 is the maintaining formula for the conflict lazy invalidation. See [2] for a list of the maintaining formulae for each[12]'s conflicts (expect for mixed invalidation that requires future operators).

Let $\mathcal{F}$ be a set of forbidden formulae characterizing all the forbidden interchanges, and let $\mathcal{A}$ denote the set of interchanges which do not satisfy any of the formulas in $\mathcal{F}$. Assume that $\sigma=a_{0}, \ldots, a_{k}$. Let $\sigma^{\prime}$ be obtained from $\sigma$ by interchanging two elements, say $a_{i-1}$ and $a_{i}$. We then say that $\sigma^{\prime}$ is 1-derivable from $\sigma$ with respect to $\mathcal{A}$ if $\left(a_{0}, \ldots, a_{i}\right) \not \forall \bigvee \mathcal{F}$. Similarly, we say that $\sigma^{\prime}$ is derivable from $\sigma$ with respect to $\mathcal{A}$ if there exist $\sigma=\sigma_{0}, \ldots, \sigma_{\ell}=\sigma^{\prime}$ such that for every $i<\ell, \sigma_{i+1}$ is 1-derivable from $\sigma_{i}$ with respect to $\mathcal{A}$. 
A TS is serializable with respect to $\mathcal{A}$ if there exists an atomic TS that is derivable from it with respect to $\mathcal{A}$. The sequence $\breve{\sigma}$ is called the purified version of TS $\sigma$ if $\breve{\sigma}$ is obtained by removing from $\hat{\sigma}$ all aborted transactions, i.e., removing the opening and closing events for such a transaction and all the read-write events by the same client that occurred between the opening and closing events. When we specify the correctness of a transactional memory implementation, only the purified versions of the implementation's transaction sequences will have to be serializable.

\section{Specification and Implementation}

Let $\mathcal{A}$ be a set of admissible interchanges which we fix for the remainder of this section. We next describe $\operatorname{Spec}_{\mathcal{A}}$, a specification of transactional memory that generates all sequences whose corresponding TSs are serializable with respect to $\mathcal{A}$. The process Spec $_{\mathcal{A}}$ is described as a fair transition system. In every step, it outputs an element in $E_{\perp}=E \cup\{\perp\}$. The sequence of outputs it generates, once the $\perp$ elements are projected away, is the set of $T S$ s that are admissible with respect to $\mathcal{A}$. $\operatorname{Spec}_{\mathcal{A}}$ uses the following data structures:

- spec_mem: array $\mathbb{N} \mapsto \mathbb{N}$ - A persistent memory. Initially, spec_mem $[i]=0$ for all $i \in \mathbb{N}$;

- $\mathcal{Q}$ : list over $E^{t} \cup \bigcup_{p}\left\{\operatorname{mark}_{p}\right\}$ - A queue-like structure, to which elements are appended, interchanged, deleted, and removed. The sequence of elements removed from this queue-like structure defines an atomic TS that can be obtained by serialization of $\operatorname{Spec}_{\mathcal{A}}$ 's output with respect to $\mathcal{A}$. For each client $p$, it is assumed that $\operatorname{mark}_{p} \notin E_{p}$ is a new symbol. Initially, $\mathcal{Q}$ is empty;

- spec_out: scalar in $E_{\perp}=E \cup\{\perp\}-$ An output variable, initially $\perp$;

- spec_doomed: array $[1 . . n] \mapsto$ bool - An array recording which pending transactions are doomed to be aborted. Initially spec_doomed $[p]=\mathrm{F}$ for every $p$.

Fig. 1 summarized the steps taken by $S_{p e c}$. The first column describes the value of spec_out with each step; it is assumed that every step produces an output. The second column describes the effects of the step on the other variables. The third column describes the conditions under which the step can be taken. The following abbreviations are used in Fig. 1

- A client $p$ is pending if spec_doomed $[p]=\mathrm{T}$ or if $\left.\mathcal{Q}\right|_{p}$ is not empty and does not terminate with $\boldsymbol{}_{p}$;

- a client $p$ is unmarked if $\left.\mathcal{Q}\right|_{p}$ does not terminate with $\operatorname{mark}_{p}$;

- a $p$-action $a$ is locally consistent with $\mathcal{Q}$ if $\left.\mathcal{Q}\right|_{p}, a$ is a prefix of some locally consistent $p$-transaction;

- a transaction $T$ is consistent with spec_mem if every $R^{t}(x, v)$ in $T$ is either preceded by some $W^{t}(x, v)$, or else $v=$ spec_mem $[x]$;

- the update of spec_mem by a transaction $T$ is $s p e c \_m e m^{\prime}$ where for every location $x$ for which $T$ has no $W^{t}(x, v)$ actions, spec_mem ${ }^{\prime}[x]=$ spec_mem $[x]$, and for every memory location $x$ such that $T$ has some $W^{t}(x, v)$ actions, spec_mem $m^{\prime}[x]$ is the value written in the last such action in $T$; 


\begin{tabular}{|c|c|c|}
\hline spec_out & other updates & conditions \\
\hline$\widehat{4} p$ & append $\boldsymbol{\triangleleft}_{p}$ to $\mathcal{Q}$ & $p$ is not pending \\
\hline$R_{p}^{t}(x, v)$ & append $R_{p}^{t}(x, v)$ to $\mathcal{Q}$ & $\begin{array}{l}p \text { is pending, unmarked and spec_doomed }[p]=\mathrm{F} \\
R(x, v) \text { is locally consistent with } \mathcal{Q}\end{array}$ \\
\hline$\overline{R_{p}^{t}(x, v)}$ & none & $p$ is pending, unmarked and spec_doomed $[p]=\mathrm{T}$ \\
\hline$W_{p}^{t}(x, v)$ & append $W_{p}^{t}(x, v)$ to $\mathcal{Q}$ & $p$ is pending, unmarked and spec_doomed $[p]=\mathrm{F}$ \\
\hline$\overline{W_{p}^{t}(x, v)}$ & none & $p$ is pending, unmarked and spec_doomed $[p]=\mathrm{T}$ \\
\hline$\% p$ & $\begin{array}{l}\text { delete } p \text { 's pending transaction from } \mathcal{Q} ; \\
\text { set spec_doomed }[p] \text { to } \mathrm{F}\end{array}$ & $p$ is pending \\
\hline$p_{p}$ & $\begin{array}{l}\text { update } s p e c \text { mem by } p \text { 's pending transaction; } \\
\text { remove } p \text {-pending transaction from } \mathcal{Q}\end{array}$ & $\begin{array}{l}p \text { has a consistent transaction at the front of } \mathcal{Q} \\
\text { that ends with } \operatorname{mark}_{p}(p \text { is pending and marked) }\end{array}$ \\
\hline$R_{p}^{n t}(x, v)$ & append $\boldsymbol{\triangleleft}_{p}, R_{p}^{t}(x, v), \boldsymbol{}_{p}$ to $\mathcal{Q}$ & $p$ is not pending \\
\hline$W_{p}^{n t}(x, v)$ & append $\boldsymbol{\triangleleft}_{p}, W_{p}^{t}(x, v), \boldsymbol{}_{p}$ to $\mathcal{Q}$ & $p$ is not pending \\
\hline$\perp$ & $\begin{array}{l}\text { set } \text { spec_doomed }_{[}[p] \text { to } \mathrm{T} ; \\
\text { delete all pending } p \text {-events from } \mathcal{Q}\end{array}$ & $p$ is pending and specdoomed $[p]=\mathrm{F}$ \\
\hline$\perp$ & apply a $\mathcal{A}$-valid transformation to $\mathcal{Q}$ & none \\
\hline$\perp$ & append $\operatorname{mark}_{p}$ to $\mathcal{Q}$ & $p$ is pending and unmarked \\
\hline$\perp$ & none & none \\
\hline
\end{tabular}

Fig. 1. Steps of $\operatorname{Spec}_{\mathcal{A}}$

- an $\mathcal{A}$-valid transformation to $\mathcal{Q}$ is a sequence of interchanges of $\mathcal{Q}$ 's entries that is consistent with $\mathcal{A}$. To apply the transformations, each $\operatorname{mark}_{p}$ is treated as if it is ${ }_{p}$.

The role of spec_doomed is to allow $S_{p e c}$ to be implemented with various arbitration policies. It can, at will, schedule a pending transaction to be aborted by setting spec_doomed $[p]$, by so "dooming" $p$ 's pending transaction to be aborted. The variable spec_doomed $_{[}[\mathrm{p}]$ is reset once the transaction is actually aborted (when $\operatorname{Spec}_{\mathcal{A}}$ outputs $\checkmark$ ). Note that actions of doomed transactions are not recorded on $\mathcal{Q}$.

We assume a fairness requirement, namely, that for every client $p=1, \ldots, n$, there are infinitely many states of $\operatorname{Spec}_{\mathcal{A}}$ where $\left.\mathcal{Q}\right|_{p}$ is empty and spec_doomed $[p]=$ F. This implies that every transaction eventually terminates (commits or aborts). It also guarantees that the sequence of outputs is indeed serializable. Note that unlike the specification described in [3] where progress can always be guaranteed by aborting transactions, here, because of the non-transactional accesses, there are cases where $\mathcal{Q}$ cannot be emptied.

While $\operatorname{Spec}_{\mathcal{A}}$ resembles its counterpart in [3], the treatment of non-transactional accesses entailed numerous changes: Roughly speaking, each transactional access is appended to the queue, and removed from it when the transaction commits, aborts, or is doomed to abort. When a transaction attempts to commit, a special marker mark $_{p}$ is appended to the queue, and, if there are admissible interchanges that move the whole transaction into the head of the queue, its events are removed from the queue and it commits. Thus, the queue never contains $>$-events, and it contains at most one mark-event. A non-transactional $p$-access $a_{p}^{n t}$ (that can only be accepted when $p$-has no pending transaction) is treated by appending $\boldsymbol{\triangleleft}_{p}, a_{p}^{t}, \boldsymbol{}_{p}$ to the queue. (Note that the non-transactional event is replaced by its transactional counterpart.) Hence, here the queue may have events. The transactions (or, rather, non-transaction) corresponding to them cannot be "doomed to abort" since such a transaction is, by definition (see below), not pending. The liveness properties require that all transaction are eventually removed from the queue. As a consequence, unlike its [3] version, $\operatorname{Spec}_{\mathcal{A}}$ does not support "eager version management" that eagerly updates the memory with every $W^{t}$-action (that doesn't 
conflict any pending transaction) which is reasonable since eager version management and the requirement to commit each non-transactional access are contradictory.

A sequence $\sigma$ over $E$ is compatible with $\operatorname{Spec}_{\mathcal{A}}$ if it can be obtained by the sequence of spec_out that $\operatorname{Spec}_{\mathcal{A}}$ outputs once all the $\perp$ 's are removed. We then have:

Claim. For every sequence $\sigma$ over $E, \sigma$ is compatible with $S_{p e c}$ iff $\hat{\sigma}$ is serializable with respect to $\mathcal{A}$.

An implementation TM: (read,commit) of a transactional memory consists of a pair of functions read: $\operatorname{pref}(T S) \times[1 . . n] \times \mathbb{N} \rightarrow \mathbb{N}$ and commit $:$ pref $(T S) \times[1 . . n] \rightarrow$ $\{a c k, e r r\}$ For a prefix $\sigma$ of a TS, $\operatorname{read}(\sigma, p, x)$ is the response (value) of the memory to an accepted $\iota R_{p}^{n t}(x) / \iota R_{p}^{t}(x)$ request immediately following $\sigma$, and $\operatorname{commit}(\sigma, p)$ is the response (ack or err) of the memory to a $\iota{ }_{p}$ request immediately following $\sigma$.

A TS $\sigma$ is said to be compatible with the memory TM if:

1. For every prefix $\eta R_{p}^{n t}(x, u)$ or $\eta R_{p}^{t}(x, u)$ of $\sigma, \operatorname{read}(\eta, p, x)=u$.

2. For every prefix $\eta{ }_{p}$ of $\sigma$, commit $(\eta, p)=a c k$.

An implementation TM: (read,commit) is a correct implementation of a transactional memory with respect to $\mathcal{A}$ if every TS compatible with $T M$ is also compatible with $\operatorname{Spec}_{\mathcal{A}}$.

\section{Verifying Implementation Correctness}

We present a proof rule for verifying that an implementation satisfies the specification Spec. The rule is adapted [7], which, in turn is based on [1]'s abstraction mapping. In addition to being case in a different formal framework, and ignoring compassion (which is rarely, if ever, used in TMs), the rule generalizes on the two given in [3] by allowing for general stuttering equivalence.

To apply the underlying theory, we assume that both the implementation and the specifications are represented as OPFSs (see [2] for details). In the current application, we prefer to adopt an event-based view of reactive systems, by which the observed behavior of a system is a (potentially infinite) set of events. Technically, this implies that one of the system variables $O$ is designated as an output variable. The observation function is then defined by $\mathcal{O}(s)=s[O]$. It is also required that the observation domain always includes the value $\perp$, implying no observable event. In our case, the observation domain of the output variable is $E_{\perp}=E \cup\{\perp\}$.

Let $\eta: e_{0}, e_{1}, \ldots$ be an infinite sequence of $E_{\perp}$-values. The $E_{\perp}$-sequence $\tilde{\eta}$ is called a stuttering variant of the sequence $\eta$ if it can be obtained by removing or inserting finite strings over $\{\perp\}$ at (potentially infinitely many) different positions within $\eta$.

Let $\sigma: s_{0}, s_{1}, \ldots$ be a computation of OPFS $\mathcal{D}$, that is, a sequence of states where $s_{0}$ satisfies the initial condition, each state is a successor of the previous one, and for every justice (weak fairness) requirement, $\sigma$ has infinitely many states that satisfy the requirement. The observation corresponding to $\sigma$ (i.e., $\mathcal{O}(\sigma)$ ) is the $E_{\perp}$ sequence $s_{0}[O], s_{1}[O], \ldots$ obtained by listing the values of the output variable $O$ in each of the states. We denote by $\operatorname{Obs}(\mathcal{D})$ the set of all observations of system $\mathcal{D}$. 
Let $\mathcal{D}_{C}$ be a concrete system whose set of states is $\Sigma_{C}$, set of observations is $E_{\perp}$, observation function is $\mathcal{O}_{C}$, initial condition is $\Theta_{C}$, transition relation is $\rho_{C}$, and justice requirements are $\cup_{f \in \mathcal{F}_{C}} \mathcal{J}(f)$. Similarly, let $\mathcal{D}_{A}$ be an abstract system whose set of states, set of observations, observation function, initial condition, transition relation, and justice requirements are $\Sigma_{A}, E_{\perp}, \mathcal{O}_{A}, \Theta_{A}, \rho_{A}$, and $\cup_{f \in \mathcal{F}_{A}} \mathcal{J}(f)$ respectively. (For simplicity, we assume that neither system contains compassion requirements.) Note that we assume that both systems share the same observations domain $E_{\perp}$. We say that system $\mathcal{D}_{A}$ abstracts system $\mathcal{D}_{C}$ (equivalently $\mathcal{D}_{C}$ refines $\mathcal{D}_{A}$ ), denoted $\mathcal{D}_{C} \sqsubseteq \mathcal{D}_{A}$ if, for every observation $\eta \in \operatorname{Obs}\left(\mathcal{D}_{C}\right)$, there exists $\widetilde{\eta} \in \operatorname{Obs}\left(\mathcal{D}_{A}\right)$, such that $\widetilde{\eta}$ is a stuttering variant of $\eta$. In other words, modulo stuttering, $\operatorname{Obs}\left(\mathcal{D}_{C}\right)$ is a subset of $\operatorname{Obs}\left(\mathcal{D}_{A}\right)$. We denote by $s$ and $S$ the states of $\mathcal{D}_{C}$ and $\mathcal{D}_{A}$, respectively.

Rule ABS-REL in Fig. 2 is a proof rule to establish that $\mathcal{D}_{C} \sqsubseteq \mathcal{D}_{A}$. The method advocated by the rule assumes the identification of an abstraction relation $R(s, S) \subseteq$ $\Sigma_{C} \times \Sigma_{A}$. If the relation $R(s, S)$ holds, we say that the abstract state $S$ is an $R$-image of the concrete state $s$.

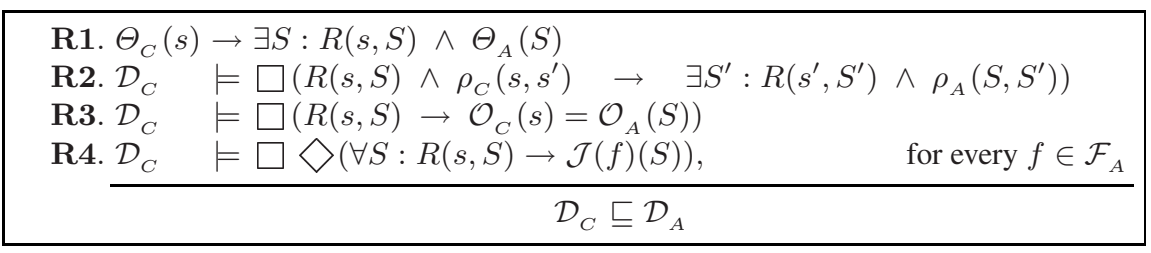

Fig. 2. Rule ABS-REL

Premise R1 of the rule states that for every initial concrete state $s$, it is possible to find an initial abstract state $S \models \Theta_{A}$, such that $R(s, S)=\mathrm{T}$.

Premise R2 states that for every pair of concrete states, $s$ and $s^{\prime}$, such that $s^{\prime}$ is a $\rho_{C}{ }^{-}$ successor of $s$, and an abstract state $S$ which is a $R$-related to $s$, there exists an abstract state $S^{\prime}$ such that $S^{\prime}$ is $R$-related to $s^{\prime}$ and is also a $\rho_{A}$-successor of $S$. Together, R1 and R2 guarantee that, for every run $s_{0}, s_{1}, \ldots$ of $\mathcal{D}_{C}$ there exists a run $S_{0}, S_{1}, \ldots$, of $\mathcal{D}_{A}$, such that for every $j \geq 0, S_{j}$ is $R$-related to $s_{j}$. Premise R3 states that if abstract state $S$ is $R$-related to the concrete state $s$, then the two states agree on the values of their observables. Together with the previous premises, we conclude that for every $\sigma$ a run of $\mathcal{D}_{C}$ there exists a corresponding run of $\mathcal{D}_{A}$ which has the same observation as $\sigma$. Premise R4 ensures that the abstract justice requirements hold in any abstract state sequence which is a (point-wise) $R$-related to a concrete computation. Here, $\square$ is the (linear time) temporal operator for "henceforth", $\diamond$ the temporal operator for "eventually", thus, $\square \diamond$ means "infinitely often." It follows that every sequence of abstract states which is $R$-related to a concrete computation $\sigma$ and is obtained by applications of premises R1 and R2 is an abstract computation whose observables match the observables of $\sigma$. This leads to the following claim which was proved using TLPVS (see Section 6):

Claim. If the premises of rule ABS-REL are valid for some choice of $R$, then $\mathcal{D}_{A}$ is an abstraction of $\mathcal{D}_{C}$. 


\section{An Example: TCC with Non-transactional Accesses}

We demonstrate our proof method by verifying a TM implementation which is essentially TCC [4] augmented with non-transactional accesses. Its specifications is given by $\operatorname{Spec}_{\mathcal{A}}$ where $\mathcal{A}$ is the admissible set of events corresponding to the lazy invalidation conflict described in Subsection 2.2 .

In the implementation, transactions execute speculatively in the clients' caches. When a transaction commits, all pending transactions that contain some read events from addresses written to by the committed transaction are "doomed." Similarly, nontransactional writes cause pending transactions that read from the same location to be "doomed." A doomed transactions may execute new read and write events in its cache, but it must eventually abort.

Here we present the implementation, and in Section 6 explain how we can verify that it refines its specification using the proof rule ABS-REL in TLPVS. We refer to the implementation as TM. It uses the following data structures:

- imp_mem: $\mathbb{N} \rightarrow \mathbb{N}$-A persistent memory. Initially, for all $i \in \mathbb{N}$, imp_mem $[i]=0$;

- cache: $\operatorname{array}[1 . . n]$ of list of $E^{t}-$ Caches of clients. For each $p \in[1 . . n]$, cache $[p]$, initially empty, is a sequence over $E_{p}^{t}$ that records the actions of $p$ 's pending transaction;

- imp_out: scalar in $E_{\perp}=E \cup\{\perp\}$ - an output variable recording responses to clients, initially $\perp$;

- imp_doomed: array $[1 . . n]$ of booleans - An array recording which transactions are doomed to be aborted. Initially imp_doomed $[p]=\mathrm{F}$ for every $p$.

$T M$ receives requests from clients, to each it updates its state, including updating the output variable imp_out, and issues a response to the requesting client. The responses are either a value in $\mathbb{N}$ (for a $\iota R^{t}$ or $\iota R^{n t}$ requests), an error $\operatorname{err}$ (for $\iota \gg$ requests that cannot be performed), or an acknowledgment $a c k$ for all other cases. Fig. 3 describes the actions of $T M$, where for each request we describe the new output value, the other updates to TM's state, the conditions under which the updates occur, and the response to the client that issues the request. For now, ignore the comments in the square brackets under the "conditions" column. The last line represents the idle step where no actions occurs and the output is $\perp$.

Comment: For simplicity of exposition, we assume that clients only issue reads for locations they had not written to in the pending transaction.

The specification of Section 3 specifies not only the behavior of the Transactional Memory but also the combined behavior of the memory when coupled with a typical clients module. A generic clients module, Clients $(n)$, may, at any step, invoke the next request for client $p, p \in[1 . . n]$, provided the sequence of $E_{p}$-events issued so far (including the current one) forms a prefix of a well-formed sequence. The justice requirement of Clients $(n)$ is that eventually, every pending transaction issues an $a c k$-ed $\iota$ or an $\iota$.

Combining modules $T M$ and Clients $(n)$ we obtain the complete implementation, defined by:

$$
\text { Imp : } \quad T M|| \operatorname{Clients(n)}
$$




\begin{tabular}{|c|c|c|c|c|}
\hline Request & imp_out & Other Updates & Conditions & Response \\
\hline$\iota \iota_{p}$ & $\mathbf{4}_{p}$ & append $\boldsymbol{\sim}_{p}$ to cache $[p]$ & {$[$ cache $[p]$ is empty] } & $a c k$ \\
\hline$\iota R_{p}^{t}(x)$ & $R_{p}^{t}(x, v)$ & append $R_{p}(x, v)$ to $\operatorname{cache}[p]$ & $\begin{array}{l}v=\text { imp_mem }[x] ; \\
{[\text { cache }[p] \text { is empty }]} \\
\text { (see comment) }\end{array}$ & imp_mem $[x]$ \\
\hline$\iota W_{p}^{t}(x, v)$ & $W_{p}^{t}(x, v)$ & append $W_{p}(x, v)$ to $\operatorname{cach} e[p]$ & {$[$ cache $[p]$ is not empty] } & $a c k$ \\
\hline$\iota \downarrow p$ & $\forall p$ & $\begin{array}{l}\text { set cache }[p] \text { to empty; } \\
\text { set imp_doomed }[p] \text { to } \mathrm{F}\end{array}$ & {$[$ cache $[p]$ is not empty] } & ack \\
\hline$\iota p_{p}$ & $p$ & $\begin{array}{l}\text { set } \text { cache }[p] \text { to empty; } \\
\text { for every } x \text { and } q \neq p \text { such that } \\
W_{p}^{t}(x) \in \text { cache }[p] \text { and } \\
R_{p}^{t}(x) \in \text { cache }[q] \\
\text { set } \text { spec_doomed }[q] \text { to } \mathrm{T} \text {; } \\
\text { update imp_mem by cache }[p]\end{array}$ & $\begin{array}{l}\text { imp_doomed }[p]=\mathrm{F} \\
{[\text { cache }[p] \text { is not empty }]}\end{array}$ & ack \\
\hline$\iota p_{p}$ & $\perp$ & none & $\begin{array}{l}\text { imp_doomed }[p]=\mathrm{T} \\
{[\text { cache }[p] \text { is not empty }]}\end{array}$ & err \\
\hline$\iota R_{p}^{n t}(x)$ & $R_{p}^{n t}(x, v)$ & none & $\begin{array}{l}v=\text { imp_mem }[x] ; \\
{[\text { cache }[p] \text { is empty }]}\end{array}$ & imp_mem $[x]$ \\
\hline$\iota W_{p}^{n t}(x, v)$ & $W_{p}^{n t}(x, v)$ & $\begin{array}{l}\text { set imp_mem }[x] \text { to } v \text {; } \\
\text { for every } q \text { such that } \\
R^{t}(x) \in \text { cache }[q] \\
\text { set imp_doomed }[q] \text { to T }\end{array}$ & {$[$ cache $[p]$ is empty] } & ack \\
\hline none & $\perp$ & none & none & none \\
\hline
\end{tabular}

Fig. 3. The actions of $T M$

where ||| denote the synchronous composition operator defined in [6]; This composition in combines several of the actions of each of the modules into one.

The actions of Imp can be described similarly to the one given by Fig. 3, where the first and last column are ignored, the conditions in the brackets are added. The justice requirements of Clients $(n)$, together with the observation that both $\iota$ and an acked $\iota>$ cause the cache of the issuing client to be emptied, imply that Imp's justice requirement is that for every $p=1, \ldots, n$, cache $[p]$ is empty infinitely many times.

The application of rule ABS-REL requires the identification of a relation $R$ which holds between concrete and abstract states. In [3], we used the relation defined by:

$$
\begin{gathered}
\text { spec_out }=\text { imp_out } \wedge \text { spec_mem }=\text { imp_mem } \\
\wedge \text { spec_doomed }=\text { imp_doomed } \\
\wedge \wedge_{p=1}^{n} \text { imp_doomed }[\mathrm{p}] \longrightarrow\left(\left.\mathcal{Q}\right|_{p}=\text { cache }[p]\right)
\end{gathered}
$$

however, there the implementation did not support non-transactional accesses. In Section 6 we provide the relation that was applied when proving the augmented implementation using TLPVS.

\section{Deductive Verification in TLPVS}

In this section we describe how we used TLPVS [11] to verify the correctness of the implementation provided in Section 5. TLPVS was developed to reduce the substantial 
manual effort required to complete deductive temporal proofs of reactive systems. It embeds temporal logic and its deductive framework within the high-order theorem prover, PVS [10]. It includes a set of theories defining linear temporal logic (LTL), proof rules for proving soundness and response properties, and strategies which aid in conducting the proofs. In particular, it has a special framework for verifying unbounded systems and theories. See [10] and [11] for thorough discussions for proving with PVS and TLPVS, respectively. In [3] we described the verification of three known transactional memory implementations with the (explicit-state) model checker TLC. This verification involved $\mathrm{TLA}^{+}[8]$ modules for both the specification and implementation, and abstraction mapping associating each of the specification's variables with an expression over the implementation's variables.

This effort has several drawbacks: The mapping does not allow for abstraction relations between states, but rather for mappings between variables. We therefore used a proof rule that is weaker than ABS-REL and auxiliary structures. For example, for $\left.\mathcal{Q}\right|_{p}=$ cache $[p]$, which cannot be expressed in $\mathrm{TLA}^{+}$, we used an auxiliary queue that can be mapped into $\mathcal{Q}$ and that records the order in which events are invoked in the implementation. And, like any other model checking took, TLC can only be used to verify small instantiations, rather than the general case. A full deductive verification requires a theorem prover.

Our tool of choice is TLPVS. Since, however, TLPVS only supports the model of PFS, we formulate OPFS in the PVS specification language. We then defined a new theory that uses two OPFSs, one for the abstract system (specification) and another for the concrete system (implementation), and proved, in a rather straightforward manner, that the rule ABS-REL is sound.

We then defined a theory for the queue-like structures used in both specification and implementation. This theory required, in addition to the regular queue operations, the definition of the projection $(\mid)$ and deletion of projected elements, which, in turn, required the proofs of several auxiliary lemmas.

Next all the components of both OPFS's defining the abstract and concrete systems were defined. To simplify the TLPVS proofs, some of the abstract steps were combined. For example, when a $\operatorname{Spec}_{\mathcal{A}}$ commits a transaction, we combined the steps of interchanging events, removing them from $\mathcal{Q}$, and setting spec_out to $\downarrow$. This restricts the set of $S p e c_{\mathcal{A}}$ 's runs but retains soundness. Formally, $T M \sqsubseteq \widehat{\operatorname{Spec}}_{\phi_{l i}}$ implies that $T M \sqsubseteq S p e c_{\phi_{l i}}$, where $\widetilde{\operatorname{Spec}}_{\phi_{l i}}$ is the restricted specification

The abstraction relation $R$ between concrete and abstract states was defined by:

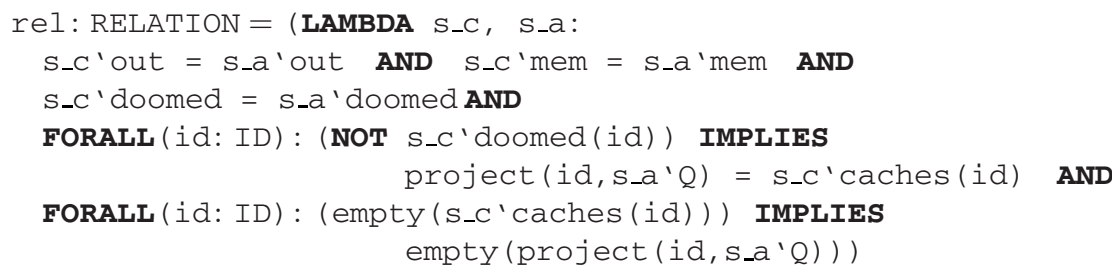

Here, s_c is a concrete state and s_a is an abstract state. The relation $R$ equates the values of out, mem and doomed in the two systems. It also states that if the transaction of a client is not doomed, then its projection on the abstract $\mathcal{Q}$ equals to the concrete 
client's cache, and if the concrete cache is empty then so is the projection of the abstract $\mathcal{Q}$ on the client's current transaction. An additional invariant, stating that each value read by a non-doomed client is consistent with the memory was also added.

In order to prove that $T M \sqsubseteq \operatorname{Spec}_{\phi_{l i}}, \mathcal{D}_{C}$ and $\mathcal{D}_{A}$ of ABS-REL were instantiated with $T M$ and $S_{p e c} c_{\phi_{l i}}$, respectively, and all the premises were verified. The proofs are in http: //cs.nyu.edu/acsys/tlpvs/tm.html.

\section{Conclusion and Future Work}

We extended our previous work on a verification framework for transactional memory implementations against their specifications, to accommodate non-transactional memory accesses. We also developed a methodology for verifying transactional memory implementations based on the theorem prover TLPVS that provides a framework for verifying parameterized systems against temporal specifications. We obtained mechanical verifications of both the soundness of the method and the correctness of an implementation which is based on TCC augmented with non-transactional accesses.

Our extension for supporting non-transactional accesses is based on the assumption that an implementation can detect such accesses. We are currently working on weakening this assumption. We are also planning to study liveness properties of transactional memory.

\section{References}

1. Abadi, M., Lamport, L.: The existence of refinement mappings. Theoretical Computer Science 82(2), 253-284 (1991)

2. Cohen, A., Pnueli, A., Zuck, L.D.: Verification of transactional memories that support nontransactional memory accesses. In: TRANSACT 2008 (February 2008)

3. Cohen, A., O'Leary, J.W., Pnueli, A., Tuttle, M.R., Zuck, L.D.: Verifying correctness of transactional memories. In: Proceedings of the 7th International Conference on Formal Methods in Computer-Aided Design (FMCAD), November 2007, pp. 37-44 (2007)

4. Hammond, L., Wong, W., Chen, M., Carlstrom, B.D., Davis, J.D., Herzberg, B., Prabhu, M.K., Wijaya, H., Kozyrakis, C., Olukotun, K.: Transactional memory coherence and consistency. In: Proc. $31^{\text {st }}$ annu. Int. Symp. on COmputer Architecture, p. 102. IEEE Computer Society, Los Alamitos (2004)

5. Herlihy, M., Moss, J.E.B.: Transactional memory: architectural support for lock-free data structures. In: ISCA 1993: Proceedings of the 20th annual international symposium on Computer architecture, San Diego, California, United States, pp. 289-300. ACM Press, New York (1993)

6. Kesten, Y., Pnueli, A.: Verification by augmented finitary abstraction. Inf. Comput. 163(1), 203-243 (2000)

7. Kesten, Y., Pnueli, A., Shahar, E., Zuck, L.D.: Network invariants in action. In: Brim, L., Jančar, P., Křetínský, M., Kucera, A. (eds.) CONCUR 2002. LNCS, vol. 2421, pp. 101-115. Springer, Heidelberg (2002)

8. Lamport, L.: Specifying Systems: The TLA ${ }^{+}$Language and Tools for Hardware and Software Engineers. Addison-Wesley, Reading (2002) 
9. Larus, J.R., Rajwar, R.: Transactional Memory. Morgan \& Claypool Publishers (2007)

10. Owre, S., Shankar, N., Rushby, J.M., Stringer-Calvert, D.W.J.: PVS System Guide. Computer Science Laboratory, SRI International, Menlo Park, CA (September 1999)

11. Pnueli, A., Arons, T.: Tlpvs: A pvs-based ltl verification system. In: Dershowitz, N. (ed.) Verification: Theory and Practice. LNCS, vol. 2772, pp. 596-625. Springer, Heidelberg (2004)

12. Scott, M.L.: Sequential specification of transactional memory semantics. In: Proc. TRANSACT the First ACM SIGPLAN Workshop on Languages, Compiler, and Hardware Suppport for Transactional Computing, Ottawa (2006) 